\title{
Croissance juvénile du chêne rouge sur des sols carbonatés à des profondeurs variables
}

\author{
J. Timbal et J. Gelpe
}

Laboratoire de Sylviculture et Ecologie, INRA, Domaine-de-l'Hermitage, Pierroton, 33610 Cestas, France

\section{Introduction}

La sensibilité du chêne rouge au calcaire est un des caractères écologiques important de l'espèce qui limite son utilisation en reboisement.

D'abord constatée empiriquement (Klejber, 1954; Polge et Zahnd, 1981), cette sensibilité au calcaire a été ensuite expérimentalement mise en évidence et précisée. Ainsi, Dewilder (1984) montre que des racines excisées de plantules de chêne rouge avaient, en présence de $\mathrm{Ca}^{2}+$ le même type d'efflux de $\mathrm{K}+$ qu'une plante typiquement calcifuge, le lupin jaune. De plus, à forte concentration de $\mathrm{Ca}^{2+}\left(25 \mathrm{mM}\right.$ de $\mathrm{CaCl}_{2}$ ), correspondaient une activité photosynthétique réduite et une croissance réduite des plantules. Nous-mêmes (Timbal et Gelpe, 1986), avons montré, par culture en pot, que cette sensibilité apparaissait dès $1 \%$ (en volume) de $\mathrm{CaCO}_{3}$ dans le substrat; sensibilité se traduisant par un aspect chlorotique des feuilles et un taux de survie et une croissance réduits.

Mais, en plus de cet aspect physiologique, la sensibilité du chêne rouge au calcaire $a$ un aspect pratique, très impor- tant pour le reboiseur: peut-on cultiver sans risque du chêne rouge sur des sols carbonatés en profondeur mais décarbonatés en surface? Pour tenter de répondre à cette question, nous avons fait germer et pousser durant deux saisons de végétation du chêne rouge sur un sol calcaire recouvert d'une couche d'épaisseur variable d'un sol non carbonaté.

\section{Matériels et Méthodes}

Glands de chêne rouge de provenance locale (artificielle), calibrés; pots de culture de grande capacité (environ $70 \mathrm{l})$ remplis d'un sol calcaire (grave calcaire) recouvert d'une couche d'épaisseur variable $(0,15,30,40 \mathrm{~cm})$ de sol non carbonaté (limon acide et désaturé); alimentation en eau non limitante.

La modalité calcaire pure a été répétée 4 fois; elle contenait 24 plants par conteneur (forte mortalité prévisible). Les autres modalités ont été répétées 16 fois et contenaient 6 plants par pot.

L'expérience s'est déroulée en serre pendant deux saisons de végétation (1986 et 1987). Mesures réalisées: la croissance (et sa décomposition en cycles) durant les deux saisons; à la fin de la deuxième année: biomasses des tiges (sans les feuilles) et des racines, après passage 
Tableau I. Mortalité et croissance.

\begin{tabular}{lccccc}
\hline & $\begin{array}{l}\text { Calcaire } \\
\text { pur }\end{array}$ & $\begin{array}{c}\text { Calcaire } \\
\text { a 15 cm }\end{array}$ & $\begin{array}{c}\text { Calcaire } \\
\text { a 30 cm }\end{array}$ & $\begin{array}{c}\text { Calcaire } \\
\text { a 40 cm }\end{array}$ & $\begin{array}{c}\text { Limons } \\
\text { purs }\end{array}$ \\
\hline Mortalité (\%) & 52,1 & 0 & 5,2 & 2,1 & 4,2 \\
Croissance totale (\%) & 17,9 & 72,1 & 103,0 & 103,2 & 107,6 \\
Diamètre au collet (mm) & $<3$ & 10,74 & 13,16 & 13,50 & 13,55 \\
Biomasses totales (g) & 5,08 & 69,04 & 120,31 & 119,70 & 115,34 \\
Rapport tiges/racines & 0,76 & 0,27 & 0,46 & 0,59 & 0,53 \\
\hline
\end{tabular}

à l'étuve à $60^{\circ} \mathrm{C}$ pendant $24 \mathrm{~h}$; teneur en éléments minéraux des feuilles (sur un échantillon moyen prélevé durant l'été chacune des deux années (analyses réalisées par le Laboratoire INRA d'Analyses végétales de Bordeaux).

Observations réalisées: débourrement au début de la $2^{\mathrm{e}}$ année; mycorhization.

De plus, le même dispositif a été installé, mais d'une manière allégée ( 2 répétitions par modalité seulement) en rhizotron (de $150 \mathrm{~cm}$ de profondeur) à raison de deux plants par rhizotron, afin de suivre la croissance racinaire.

\section{Résultats}

Dans toutes les modalités (y compris calcaire pur) les racines ont pénétré dans la couche calcaire et y ont été naturellement mycorhizées.

A la surface des pots, des carpophores sont apparus qui appartenaient essentiellement à l'espèce Laccaria laccata.

L'influence du calcaire sur la survie et la croissance du chêne rouge ne se fait sentir que si la couche non carbonatée recouvrant le sol calcaire a au moins $30 \mathrm{~cm}$ d'épaisseur. Au-delà, la croissance observée est "normale" (pas de différence significative avec le témoin "limons purs".

Cette sensibilité se traduit par: 1) une forte mortalité au stade semis $(52 \%$ en moyenne sur calcaire pur) (Tableau I); 2) une croissance réduite (surtout la $2^{\mathrm{e}}$ année) des survivants, la réduction de la croissance en hauteur étant due à une réduction du taux de polycyclisme et de la longueur moyenne de chaque cycle; 3 ) une biomasse totale réduite, avec une réduction affectant surtout les parties aériennes (réduction du rapport tiges/ racines); 4) un débourrement, en moyenne plus tardif (mais la différence est faible) dans les modalités où le calcaire est à faible profondeur que dans les autres; 5) au niveau foliaire par des symptômes de chlorose: feuilles de couleur jaunâtre et petites (surtout en largeur) et une composition minérale modifiée: forte teneur en $\mathrm{Ca}$ (ce qui est logique) (Salsac, 1980) et en fer (ce qui est étonnant) déficience en $\mathrm{N}$ et $\mathrm{P}$, et des carences nettes en $\mathrm{Mn}$ et $\mathrm{Zn}$ (Bonneau, 1986).

\section{Discussion et Conclusions}

L'expérimentation menée a permis de confirmer la sensibilité du chêne rouge au calcaire et surtout d'en préciser les modalités sur la croissance: fort taux de mortalité au stade semis et forte réduction de la croissance en hauteur par réduction du taux de polycyclisme et de la longueur moyenne des cycles.

De plus, laugmentation de biomasse, bien qu'également réduite, se fait davantage au profit des parties racinaires et cela davantage par augmentation de leur dia- 
mètre que de leur nombre (observation après déterrage).

Sur le plan de la nutrition minérale, les résultats des analyses foliaires sont conformes à ce que l'on sait de la physiologie des plantes calcifuges et de la chlorose (Salsac, 1980; Ghorbal, 1979; Berang et Steiner, 1980) sauf en ce qui concerne le fer dont la forte teneur dans les modalités avec calcaire pur ou à faible profondeur est surprenante et contraire à ce que l'on sait de la chlorose ferrique (Berang et Steiner, 1980). Ce dernier résultat mérite d'être confirmé et s'il l'était, il serait nécessaire de déterminer la forme et la localisation précise de ce fer au niveau cellulaire et des différentes parties.

Des observations plus fines sur la mycorhization qui se réalise dans les deux types de substrat seraient également intéressantes à réaliser.

Sur le plan pratique, le seul qui intéresse le sylviculteur, ces résultats sont encourageants dans la mesure où ils laissent penser que la culture du chêne rouge est possible sur des sols décarbonatés en surface sur au moins $30 \mathrm{~cm}$. II est d'ailleurs intéressant de constater que ce seuil de $30 \mathrm{~cm}$ est le même que celui mis en évidence par Kleiber (1954) sur des arbres isolés et des plantations (jusqu'à 50 ans) de la région de Schonberg (S.O. de Fribourg, Allemagne). Une telle obser- vation mériterait une confirmation expérimentale avec, en particulier, un diagnostic foliaire suivi.

\section{Références}

Berang P. \& Steiner K. (1980) Resistance of pin oak (Quercus palustris) progenies to iron chlorosis. J. Am. Soc. Hortic. Sci. 105, 519-522

Bonneau M. (1986) Cours ENGREF de pédologie forestière, $2^{\circ}$ partie, ch. VI: Le diagnostic foliaire. Doc. offset ENGREF, pp. 58

Dewilder R. (1984) Quelques aspects anatomiques et physiologiques de la racine primaire de chêne rouge d'Amérique (Quercus rubra L.). DEA Thesis, Université de Bordeaux I, France

Ghorbal M.H. (1979) Absorption du calcium, localisation et rôle dans la perméabilité membranaire, relations avec le caractère calcicole ou calcifuge. Ph.D. Thesis, Université de Montpellier, France

Kleiber H. (1954) Roteiche und kalk. Allg. Forstz. 33/34, 353-354

Polge A. \& Zahnd E. (1981) Le chêne rouge d'Amérique en Alsace. Etudes des relations entre les caractéristiques stationnelles et les performances de croissance quantitatives et qualitatives. Rev. For. Fr. 33, 195-216

Salsac L. (1980) L'absorption du calcium par les racines des plantes calcicoles et calcifuges. Sci. Sol. 1, 445-4777

Timbal J. \& Gelpe J. (1986) Action du calcaire sur la germination et la croissance du chêne rouge d'Amérique (Quercus rubra L.). Ann. Sci. For. $43,397-402$ 